\title{
A simple oligonucleotide biochip capable of rapidly detecting known mitochondrial DNA mutations in Chinese patients with Leber's hereditary optic neuropathy (LHON)
}

\author{
Wei-Dong Du ${ }^{\mathrm{a}, \mathrm{f}, *}$, Gang Chen ${ }^{\mathrm{a}, \mathrm{b}}$, Hui-Min $\mathrm{Cao}^{\mathrm{b}}$, Qing-Hui Jin ${ }^{\mathrm{b}}$, Rong-Feng Liao ${ }^{\mathrm{c}}$, Xiang-Cheng $\mathrm{He}^{\mathrm{d}}$, \\ Da-Ben Chen ${ }^{\mathrm{e}}$, Shu-Ren Huang ${ }^{\mathrm{d}}$, Hui Zhao ${ }^{\mathrm{b}}$, Yong-Mei Lv ${ }^{\mathrm{a}}$, Hua-Yang Tang ${ }^{\mathrm{a}}$, Xian-Fa Tang ${ }^{\mathrm{a}}$, \\ Yong-Qing Wang ${ }^{\mathrm{d}}$, Song Sun ${ }^{\mathrm{e}}$, Jian-Long Zhao ${ }^{\mathrm{b}, *}$ and Xue-Jun Zhang ${ }^{\mathrm{a}}$ \\ ${ }^{a}$ Key Lab of Gene Resource Utilization for Severe Hereditary Diseases of Ministry of Education of China \& Key \\ Lab of Genome Research of Anhui Province, Anhui Medical University, 230032 Hefei, China \\ ${ }^{\mathrm{b}}$ Shanghai Institute of Microsystem and Information Technology, Chinese Academy of Sciences, 200050 Shanghai, \\ China \\ ${ }^{\mathrm{c}}$ Department of Ophthalmology, the First Affiliated Hospital of Anhui Medical University, 230032 Hefei, China \\ ${ }^{\mathrm{d}}$ Ophthalmology Hospital, Red Cross Association of Anhui Province, 230022 Hefei, China \\ e Department of Ophthalmology, University of Science and Technology of China, 230026 Hefei, China \\ ${ }^{\mathrm{f}}$ Sektion Experimentelle Anaesthesiologie, Universitaetsklinikum Ulm, Steinhoevelstrasse 9, 89075 Ulm, Germany
}

\begin{abstract}
Leber's hereditary optic neuropathy (LHON) is a maternally transmitted disease. Clinically, no efficient assay protocols have been available. In this study, we aimed to develop an oligonucleotide biochip specialized for detection of known base substitution mutations in mitochondrial DNA causing LHON and to investigate frequencies of LHON relevant variants in Anhui region of China. Thirty-two pairs of oligonucleotide probes matched with the mutations potentially linked to LHON were covalently immobilized. Cy5-lablled targets were amplified from blood DNA samples by a multiplex PCR method. Two kinds of primary mutations $11778 \mathrm{G}>\mathrm{A}$ and $14484 \mathrm{~T}>\mathrm{C}$ from six confirmed LHON patients were interrogated to validate this biochip format. Further, fourteen Chinese LHON pedigrees and twenty-five unrelated healthy individuals were investigated by the LHON biochip, direct sequencing and pyrosequencing, respectively. The biochip was found to be able efficiently to discriminate homoplasmic and heteroplasmic mtDNA mutations in LHON. Biochip analysis revealed that twelve of eighteen LHON symptomatic cases from the 14 Chinese pedigree harbored the mutations either $11778 \mathrm{G}>\mathrm{A}, 14484 \mathrm{~T}>\mathrm{C}$ or $3460 \mathrm{G}>\mathrm{A}$, respectively, accounting for $66.7 \%$. The mutation $11778 \mathrm{G}>\mathrm{A}$ in these patients was homoplasmic and prevalent $(55.5 \%, 10$ of 18 cases). The mutations 3460G $>$ A and 3394T $>$ C were found to co-exist in one LHON case. The mutation 13708G $>$ A appeared in one LHON pedigree. Smaller amount of sampling and reaction volume, easier target preparation, fast and high-throughput were the main advantages of the biochip over direct DNA sequencing and pyrosequencing. Our findings suggested that primary mutations of $11778 \mathrm{G}>\mathrm{A}, 14484 \mathrm{~T}>\mathrm{C}$ or $3460 \mathrm{G}>\mathrm{A}$ are main variants of mtDNA gene leading to LHON in China. The biochip would easily be implemented in clinical diagnosis.
\end{abstract}

Keywords: Oligonucleotide biochip, mitochondrial DNA, point mutation, Leber's hereditary optical neuropathy

*Corresponding authors. Tel.: +49731 500600 80; Fax: +49 731500600 82; E-mail: weidongdu@hotmail.com \& jlzhao@mail. sim.ac.cn.

\section{Introduction}

Leber's hereditary optic neuropathy (LHON, OMIM \#535000) is a maternally transmitted disease characterized by acute or subacute visual loss predominantly af- 
fecting young men $[1,2]$. So far, more than 30 kinds of mtDNA mutations associated with the pathogenesis of LHON have been identified (http://www.mitomap.org). The LHON mutations are classified as primary and secondary mutations. The majority ( $>95 \%$ ) of LHON cases are known to harbor one of the three primary point mitochondrial DNA (mtDNA) mutations: $3460 \mathrm{G}$ $>\mathrm{A}(\mathrm{A} 52 \mathrm{~T}, \mathrm{ND} 1), 11778 \mathrm{G}>\mathrm{A}(\mathrm{R} 340 \mathrm{H}, \mathrm{ND} 4)$, and $14484 \mathrm{~T}>\mathrm{C}(\mathrm{M} 64 \mathrm{~V}, \mathrm{ND6})$, all of which affect genes encoding complex I subunits of the respiratory chain [2] and are usually screened in any suspected LHON patients. Secondary mutations do not allow proving the clinical diagnosis of LHON; their presences increase the probability and modulate the phenotype of LHON [3]. In addition, the mutations 3376G $>\mathrm{A}$ in the MTND1, 13045A $>\mathrm{C}$ in the MTND5 and $7444 \mathrm{G}>\mathrm{A}$ in the MTCO1 associate with overlap syndromes of both LHON and either Mitochondrial Encephalomyopathy, Lactic Acidosis, and Stroke-like episodes (MELAS) [4], or MELAS/Leigh's Syndrome (LS) [5], or Sensorineural Hearing Loss (SNHL) [6], respectively. The pathogenesis of LHON remains unclear. Deficiency in respiratory chain function and reactive oxygen species (ROS) resulting from mutations in mtDNA are believed to play a pivotal role in the pathophysiology of the disease [7]. Clinically, although the primary etiological factor of LHON is an mtDNA mutation, the presence of a primary mtDNA mutation does not necessarily lead to visual loss. A small number of patients lacking any of the three primary mutations have been reported [8]; in these cases, the segregation of rare pathogenic mtDNA mutations has been observed, making genetic counseling of a patient and his family or confirming a specific diagnosis a great challenge. The marked incomplete penetrance and gender bias indicate that additional genetic (nuclear or mitochondrial) and epigenetic factors may also be involved [9].

The principal difficulty for clinicians in clinical diagnosing of mtDNA relevant diseases is in that either the patients with mtDNA disease rarely have a classic phenotype or mtDNA falls into the differential diagnosis of other clinical syndromes [10]. Molecular genetic studies of mtDNA are helpful in the understanding and classification of the mitochondrial diseases. To date, a variety of single nucleotide polymorphisms (SNP) typing technologies have been broadly applied, for instance, restriction fragment length polymorphism analysis (RFLP), single strand conformation polymorphism (SSCP), real-time polymerase chain reaction, DNA sequencing, denaturing gradient gel electrophore- sis, electrochemical and microgravimetric measurements [11], minisequencing [12], pyrosequencing [13] and biochip [14]. High throughput biochips are initially developed to analyze the expression profiling of RNA transcripts in parallel and then adapted to analysis of the genome to study DNA sequence content. By employing array-based technologies, dramatic discoveries of key variants involved in human multiple complex diseases and related traits have been reported [15], rooting in the biology of these disorders and opening up new accesses for investigators to address novel molecular pathways that were not previously linked to or thought in relation with these diseases $[14,16]$. With the advent of an increasing range of new mtDNA mutations identified and the requirement for fast molecular defining, there is a need in the molecular diagnostic palette to detect clinically relevant levels of mutations in a reliable, time- and cost-effective manner. Currently, regardless of high density of biochips, functional or nosological biochips that specially focus on dozens or hundreds of features interested have been under development, making it more efficient and effective in discriminating valuable molecules, offering clinical diagnosis, and guiding individual treatments.

In this study, we developed a biochip based method for simultaneous screening of 32 known base substitution mutations that may cause LHON, and evaluated the feasibility of this biochip format in diagnostics of LHON mutations in the gmtDNA gene.

\section{Patients and methods}

\subsection{Patients and mtDNA samples}

Six clinical confirmed LHON cases with the known primary mutations $1778 \mathrm{G}>\mathrm{A}$ (4 cases) or $14484 \mathrm{~T}>$ C ( 2 cases), eighteen affected patients, 52 unaffected family members from fourteen LHON pedigrees, and 25 unrelated control individuals were collected during June 2005 and May 2006 in Anhui region of China. Consensus diagnosis of LHON was performed by two experienced ophthalmologists based on clinical findings. A bilateral vision impairment leading to blindness was present in all the $18 \mathrm{LHON}$ patients. Gender ratio of the patients was 2.6:1 (13 males and 5 females). Mean disease onset age was 23 years \pm 11 . Informed consents were obtained from all the participants. This study was approved by the ethics committee for genome research of the Anhui Medical University of China. Mitochondrial DNAs of peripheral blood samples from 


\begin{tabular}{|c|c|c|c|c|c|}
\hline \multirow{17}{*}{ 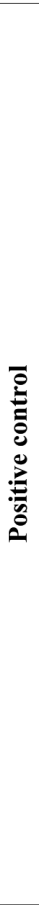 } & $3394 \mathrm{~T}$ & $3394 C$ & \multirow{17}{*}{ 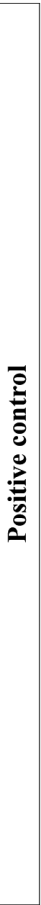 } & $11719 \mathrm{G}$ & $11719 \mathrm{~A}$ \\
\hline & $3460 \mathrm{G}$ & $3460 \mathrm{~A}$ & & $11778 \mathrm{G}$ & $11778 \mathrm{~A}$ \\
\hline & $3635 \mathrm{G}$ & $3635 \mathrm{~A}$ & & $13708 \mathrm{G}$ & $13708 \mathrm{~A}$ \\
\hline & $3733 \mathrm{G}$ & $3733 \mathrm{~A}$ & & $13730 \mathrm{G}$ & 13730A \\
\hline & $4136 \mathrm{~A}$ & $4136 \mathrm{G}$ & & $14459 \mathrm{G}$ & 14459A \\
\hline & $4160 \mathrm{~T}$ & $4160 \mathrm{C}$ & & $14482 C$ & $14482 \mathrm{~A}$ \\
\hline & $4171 \mathrm{C}$ & 4171A & & $14482 \mathrm{C}$ & $14482 \mathrm{G}$ \\
\hline & $4216 \mathrm{~T}$ & $4216 \mathrm{C}$ & & $14484 \mathrm{~T}$ & $14484 C$ \\
\hline & $4435 \mathrm{~A}$ & $4435 \mathrm{G}$ & & $14495 \mathrm{~A}$ & $14495 \mathrm{G}$ \\
\hline & $4640 \mathrm{C}$ & $4640 \mathrm{~A}$ & & $14498 \mathrm{~T}$ & $14498 \mathrm{C}$ \\
\hline & 4917A & $4917 \mathrm{G}$ & & $14568 \mathrm{C}$ & $14568 \mathrm{~T}$ \\
\hline & $5244 \mathrm{G}$ & 5244A & & $14596 \mathrm{~A}$ & 14596T \\
\hline & $7444 \mathrm{G}$ & 7444A & & $14693 \mathrm{~A}$ & $14693 \mathrm{G}$ \\
\hline & $9738 \mathrm{G}$ & $9738 \mathrm{~T}$ & & $15257 \mathrm{G}$ & $15257 \mathrm{~A}$ \\
\hline & $9804 \mathrm{G}$ & 9804A & & $15812 \mathrm{G}$ & $15812 \mathrm{~A}$ \\
\hline & $10663 \mathrm{~T}$ & $10663 \mathrm{C}$ & & $15951 \mathrm{~A}$ & $15951 \mathrm{G}$ \\
\hline & $11696 \mathrm{G}$ & $11696 \mathrm{~A}$ & & Negat & ontrol \\
\hline
\end{tabular}

Fig. 1. Layout of LHON biochip format. A mixed solution of all the probes was used as an internal positive control (P) and spotted in a column on the left side of the biochip layout. Spotting solution was used as background and spotted in the right bottom of the biochips (N).

LHON symptomatic cases, asymptomatic maternal relatives and unrelated population-matched controls were prepared by using FlexiGene DNA kit (Qiagen, Shanghai, China) according to the manufacturer's protocol.

\subsection{Design of oligonucleotide probes}

Thirty-two known mutation sites associated with LHON (Supplementary Table 1) were selected according to the MITOMAP database (http://www.mitomap. org) and human mitochondrial DNA revised Cambridge reference sequence $[17,18]$. Thirty-two pairs of allelespecific probes were designed by Primer Premier (version 5.0, Premier Biosoft international, Palo Alto, CA) and were synthesized by TaKaRa Biotechnology Inc. (Dalian, China). Each pair of the probes specific for a given mutation site shared an identical sequence with the exception of a single base substitution near the middle of the probe sequence. All the probes contained a C6 amino linker at their 5'-ends for covalent attachment onto aldehyde-coated slides. A spacer arm with a 15 mer-poly $(\mathrm{T})$ oligonucleotide was inserted between the probe sequence and the amino linker molecule to decrease steric interference on the biochips. The probe sequences were listed in Supplementary Table 2.

\subsection{Slides and biochip manufacture}

Aldehyde-coated glass slides were purchased from CEL Associates Inc. (Houston TX, USA). The biochip manufacture was performed as described previously [19]. Briefly, the thirty-two pairs of the allele specific probes were prepared in a final concentration of $12.5 \mathrm{pM}$ by a spotting solution (TeleChem International Inc., Sunnyvale, CA, USA) and robotically printed onto the activated glass slides $(0.5 \mathrm{nl} / \mathrm{spot})$ by means of a PixSys 7500 arrayer and a CMP3 spotting pin $(150 \mu \mathrm{m}$, Cartesian Technologies, Irvine, CA, USA). Three replicate spots of each probe were aligned in the same row of the biochips. A mixed solution from all the probes was used as an internal positive control (P) and spotted in a column on the left side of the biochip layout. Spotting solution was printed as a background $(\mathrm{N})$ in the bottom right hand corner of the biochip layout (Fig. 1). After the spotting procedure, the biochips were immobilized in $70 \%$ humidity environment at $25^{\circ} \mathrm{C}$ for 48 hours and stored at $4^{\circ} \mathrm{C}$.

\subsection{Multiplex PCR amplification of mtDNA targets}

Eleven pairs of PCR primers were synthesized by Sangon Biological Engineering and Technology and 
Service Co. Ltd (Shanghai, China). The primer sequences were listed in Supplementary Table 3. Amplifications of Cy5-lablled mtDNA targets containing the above-mentioned 32 mutation sites were achieved by three multiplex PCR reactions with a multiplex PCR Kit (TaKaRa Biotechnology, Inc., Dalian, China). Each PCR mixture $(25 \mu \mathrm{l})$ contained 100ng of DNA, 1xPCR buffer, $1.5 \mathrm{mM} \mathrm{MgCl} 2,200 \mu \mathrm{M}$ of each deoxynucleoside triphosphate (Boehringer Mannheim, Mannheim, Germany), 32-400 nM of each primer, and 1.5U of Taq DNA polymerase. The PCR thermocycling condition was as follows: $94^{\circ} \mathrm{C}$ for $5 \mathrm{~min} ; 35$ cycles with denaturizing at $94^{\circ} \mathrm{C}$ for $30 \mathrm{~s}$, annealing at $60^{\circ} \mathrm{C}$ for $60 \mathrm{~s}$, and elongation at $72^{\circ} \mathrm{C}$ for $90 \mathrm{~s}$; followed by a final elongation step at $72^{\circ} \mathrm{C}$ for $10 \mathrm{~min}$ in a Programmable Thermal Controller (MJ Research Inc.). PCR products were subjected to a $2.0 \%$ (wt/vol) agarose gel electrophoresis.

\subsection{Biochip assays for the LHON patients with mtDNA mutations}

Cy5-labelled targets from LHON cases, family members and the controls were respectively amplified by the multiplex PCR method. Hybridization was performed as described previously [19]. Briefly, the three unpurified Cy5-labelled PCR products were pooled. Five $\mu 1$ of the pooled target was mixed with $10 \mu \mathrm{l}$ DIG Easy Hyb solution (Roche Molecular Biochemicals, Laval, Quebec, Canada), then denatured at $95^{\circ} \mathrm{C}$ for $5 \mathrm{~min}$, and immediately quenched on ice for $5 \mathrm{~min}$. The hybridization solution was applied on the biochips and covered with glass coverslips. Hybridization was performed for 1 hour at $35^{\circ} \mathrm{C}$ in a humidity chamber. The biochips were washed once in 2 XSSC- $0.1 \%$ SDS solution at $30^{\circ} \mathrm{C}$ for $10 \mathrm{~min}$, followed by additional wash in $0.2 \mathrm{xSSC}-0.1 \% \mathrm{SDS}$ solution at $30^{\circ} \mathrm{C}$ for $5 \mathrm{~min}$ to diminish unspecific binding, and then dried with a stream of nitrogen. The fluorescence signals from all the spots on the biochips were captured by a fluorescence signal reader (GenePix 4000B, Axon Instruments, Inc.) at wavelength $635 \mathrm{~nm}$ for Cy5. Average fluorescence intensities from the three spots at each concentration were quantified with an array acquisition and analysis software (GenePix Pro 4.0). To discriminate genotype at a given site and to minimize the bench affect between experiments, a diagnostic factor of 3 from the ratio between wild type (wt) and mutation (mut) was used [20]. Sample with the factor over 3 was referred to as a wild type and the factor below 0.3 was interpreted as a homoplasmic mutant. The mutation indicated a heteroplasmy if the factor varied between 0.3 and 3 .

\subsection{Genotyping by pyrosequencing}

To confirm the mutations $11778 \mathrm{G}>\mathrm{A}, 14484 \mathrm{~T}>$ C, 3460G $>$ A and 13708G $>$ A in the LHON cases determined by the biochip, pysequencing was performed. The forward and reverse PCR primers and internal sequencing primer were designed using Pyrosequencing Assay Design Software 1.0 Version (Biotage, Uppsale. Sweden) and were synthesized by Sangon Biological Engineering and Technology and Service Co. Ltd (Shanghai, China). The primer sequences were showed in Supplementary Table 4. The singleplex PCR for individual mutation was performed in a $50 \mu \mathrm{l}$ reaction volume containing $100 \mathrm{ng}$ of DNA, $0.2 \mu \mathrm{M}$ each of forward and reverse oligonucleotide PCR primers, $0.2 \mathrm{mM}$ dNTP deoxynucleotide triphosphates, 1x PCR buffer, and $2.5 \mathrm{mM} \mathrm{MgCl} 2$ and 1.0 unit Taq DNA polymerase (AmpliTaq Gold DNA polymerase, Applied Biosystems, CA, USA). PCR reactions were amplified at $95^{\circ} \mathrm{C} 5 \mathrm{~min} ; 95^{\circ} \mathrm{C} 15 \mathrm{~s}, 57^{\circ} \mathrm{C}, 30 \mathrm{~s}, 72^{\circ} \mathrm{C}$ $15 \mathrm{~s}, 50$ cycles, and $72^{\circ} \mathrm{C} 5 \mathrm{~min}$. Templates for each pyrosequencing assay were purified from a $40 \mu \mathrm{l} \mathrm{PCR}$ reaction volume. Genotyping was performed at room temperature using an automated pyrosequencer (PSQ 96MA Pyrosequencer, Biotage, Uppsala, Sweden) and a PSQTM96 Pyro Gold reagent kit according to our previous report [21].

\subsection{Sequencing analysis}

Likewise, the genotyping results of the biochips in the LHON patients and the controls were also validated by direct sequencing. Eleven individual PCR products were amplified by the identical PCR primers used in biochip assay. Forward primers in PCR reactions were used for sequencing. The direct sequencing was performed at Shanghai Invitrogen Biotechnology Co., Ltd with an ABI PRISM dye terminator cycle sequencing ready reaction kit (Perkin-Elmer, Applied Biosystems, Foster City, California) and an ABI 377A automated DNA sequencer. The sequences were analyzed with Sequence Navigator software (Perkin-Elmer, Applied Biosystems).

\section{Results and discussion}

\subsection{Validation of biochip detection for the primary mitochondrial LHON mutations}

Here, we established a simple oligonucleotide biochip format and validated the potential of this 


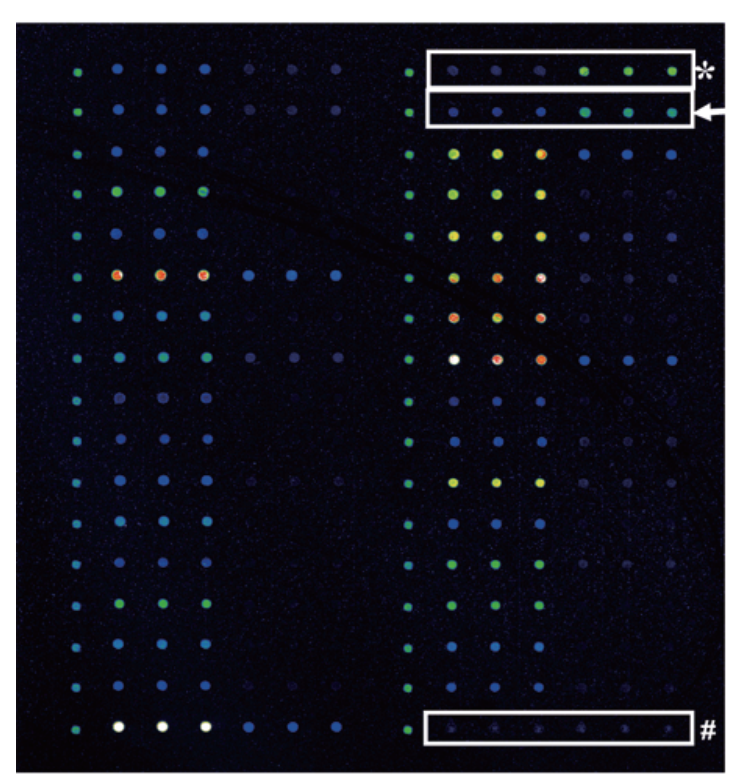

A
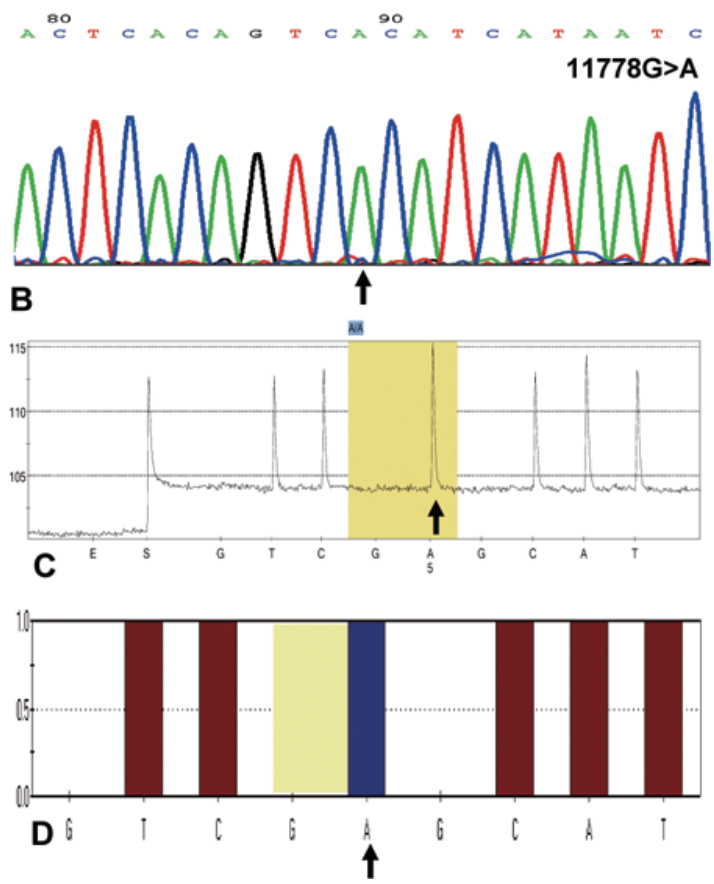

Fig. 2. Detection of $11778 \mathrm{G}>$ A homoplasmic mutation in a patient with LHON by the biochip (A), direct sequencing (B) and pyrosequencing (C). The arrow in the figure A indicated where the mutation $11778 \mathrm{G}>$ A happened. mtDNA mutation 11719 was localized in the box marked with an asterisk in the top right hand corner of the biochip layout. The box with \# number in the bottom right hand corner of the biochip denoted negative control. A histogram of homoplasmic $11778 \mathrm{G}>$ A detection of pyrosequencing assay was shown as a reference (D).

biochip in the interrogation of two known primary mutations $(11778 \mathrm{G}>\mathrm{A}$ and $14484 \mathrm{~T}>\mathrm{C})$ from six confirmed LHON DNA samples. We obtained 11 amplicons containing 32 selected mutations by a multiplex PCR method. Targeting of neighboring mutations was achieved with the aid of either $H$ or $L$ chain probes. Five $\mu \mathrm{l}$ of unpurified multiplex PCR pooled solution was used for on-chip hybridization of the 32 pairs of the features. We found that only the probes that perfectly matched the corresponding targets were successfully detected. The primary mutations were interpreted by the biochip to be homoplasmic with a wt/mut ratio factor of 0.21 at the sites of 11778 (Fig. 2) and 0.01 at 14484 (Fig. 3). The wt/mut ratio values in these cases were all over 3 at the internal reference wild-type sites on the biochip, ranging from 4.5 to 368.3 . The panel of the 32 known mutations in all the unrelated controls showed homoplasmic wild-type sequences entirely. The ranges of wt/mut ratio values in the controls varied between 4.2 and 357.4. The fluorescence signal resolution of each spot on the biochips appeared high enough for discrimination of homoplasmic and heteroplasmic mtDNA mutations individually. The biochip met the need of LHON mutation assay.

\subsection{Comparison of three SNP typing methodologies in detecting LHON mutations}

In this study, the mutations and their homoplasmic or heteroplasmic status in LHON by the biochip were further confirmed by both direct sequencing and pyrosequencing. In this study, we amplified and purified 11 individual PCR fragments and performed the subsequent direct sequencings for each sample to achieve the 32 mutations. Direct sequencing by the Sanger method is broadly used for mutation detection in genes, being able to span longer DNA stretch, to offer reading of flanking sequences near the SNPs in question and to reveal any novel and known rare disease causing mutations that have not been reported as having demonstrated risk in the population. However, analysis of $\mathrm{mtD}$ NA variation with this method does not allow accurate quantification of the components of mtDNA mixtures [10]. The effort that it adds achieves little clinical value for whole-mtDNA genome variant analysis, as a result of being laborious and time consuming. Pyrosequencing technique, on the other hand, can be applied for genotyping and sequence determination in mixtures of mtDNA [12], but the attempt to comprehensively target the important informative variants ranging the 


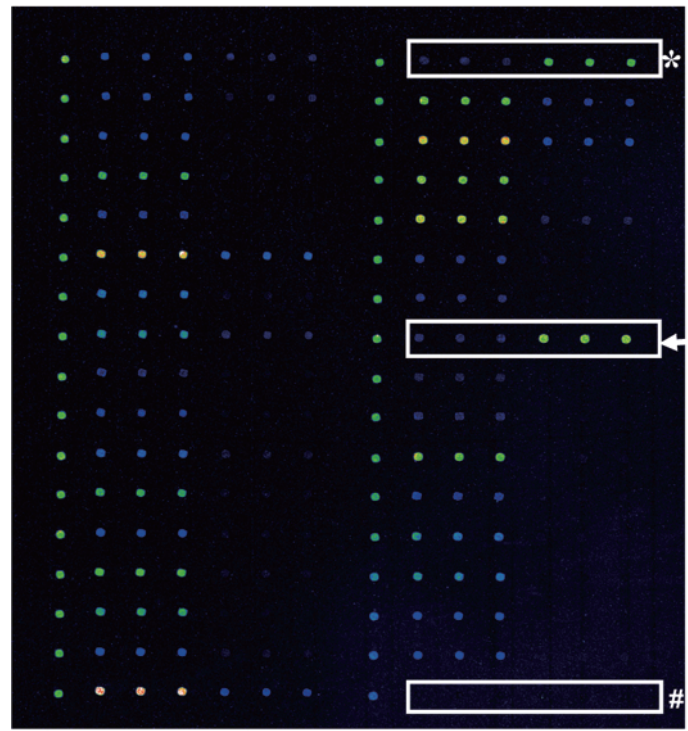

A

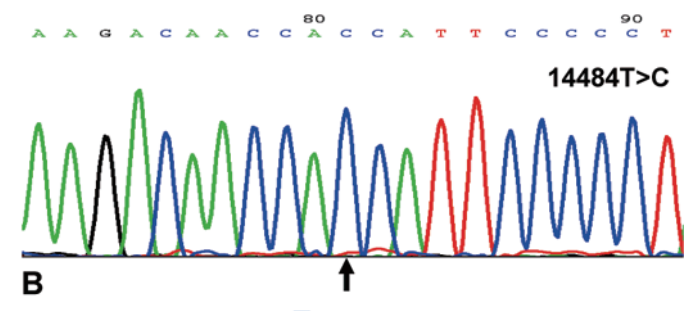

ब西
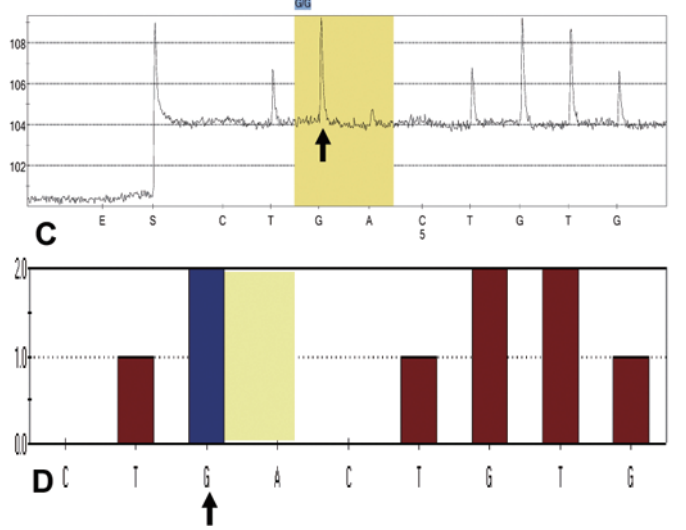

Fig. 3. Detection of $14484 \mathrm{~T}>\mathrm{C}$ homoplasmic mutation in a patient with LHON by the biochip (A), direct sequencing (B) and pyrosequencing (C). Primary 14484T $>\mathrm{C}$ homoplasmic mutation was indicated in the box with an arrow and the mutation 11719 was shown with an asterisk in the figure A. A histogram of homoplasmic 14484T $>$ C detection of pyrosequencing assay was shown as a reference (D).

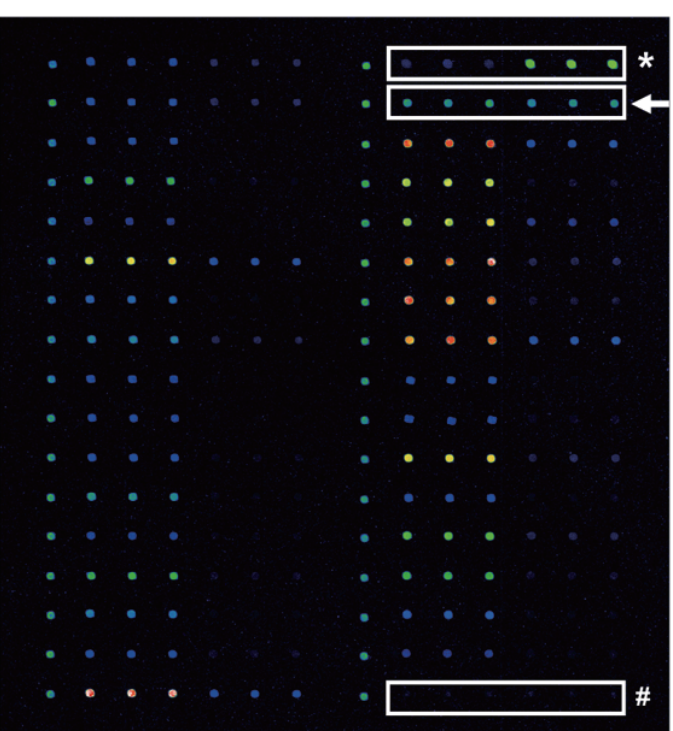

A

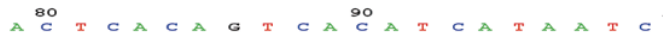

11778G $>A$
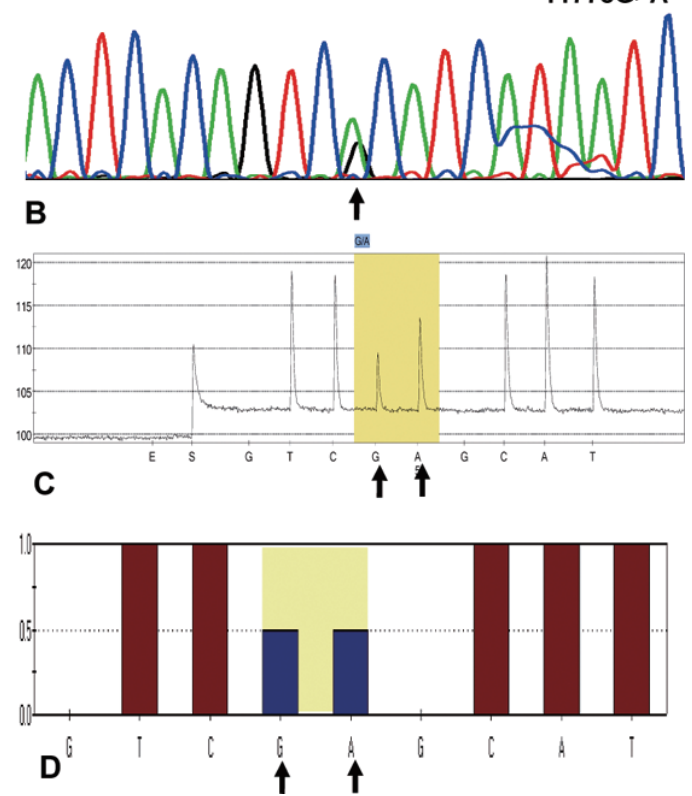

Fig. 4. Detection of primary 11778 heteroplasmic mutation in a patient with LHON by the biochip (A), direct sequencing (B) and pyrosequencing (C). The wt/mut ratio was 1.11 at the site of 11778 shown by the biochip. The mutation 11719 was indicated in the box with an asterisk in the top right hand corner of the biochip. A histogram of 11778 heteroplasmic mutation of pyrosequencing assay was shown as a reference (D). 


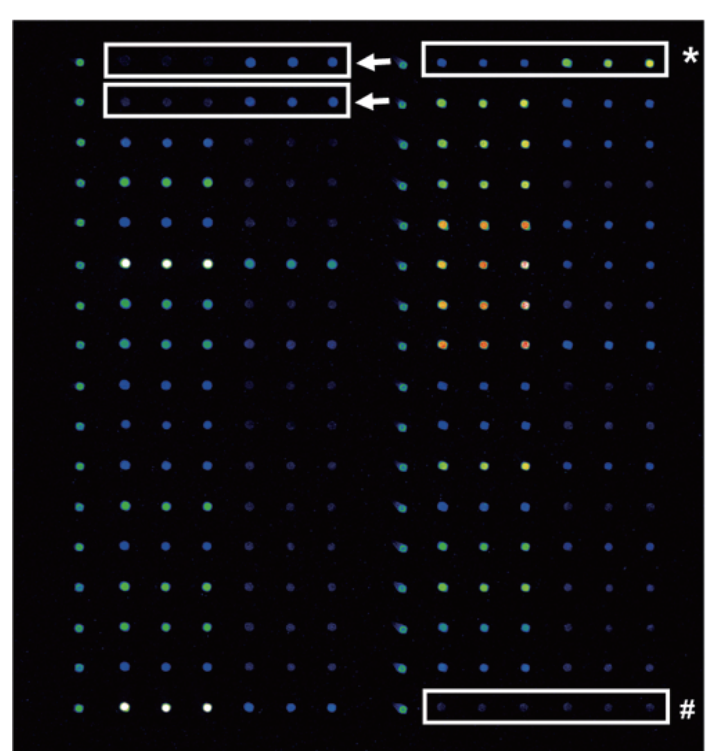

A
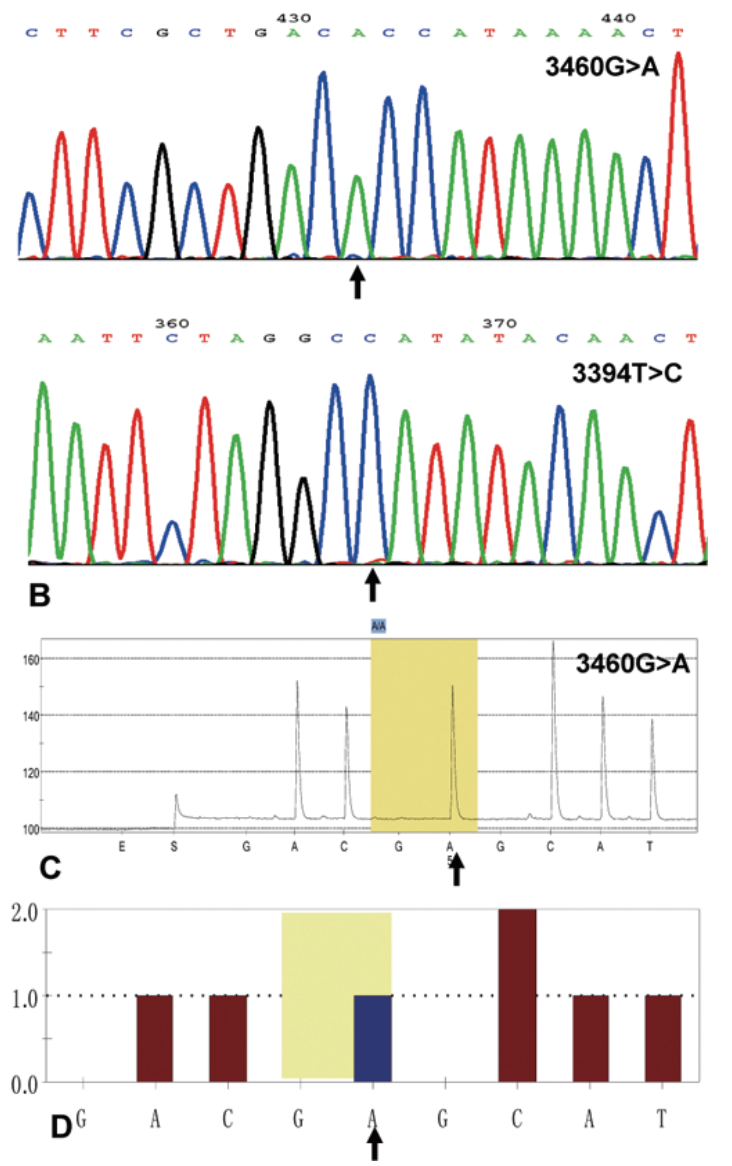

Fig. 5. Co-existence of $3460 \mathrm{G}>\mathrm{A}$ and $3394 \mathrm{~T}>\mathrm{C}$ causal homoplasmic mutations in a LHON patient detected by the biochip (A), direct sequencing $(\mathrm{B})$ and pyrosequencing $(\mathrm{C})$. The first arrow in the upper left corner of the biochip layout indicated the mutation 3394T $>\mathrm{C}$ and the second one denoted the mutation 3460G $>$ A. mtDNA mutation 11719 was localized with an asterisk. The wt/mut ratios were 0.24 at the site of mtDNA 3460G $>$ A and 0.16 at mtDNA 3394T $>$ C, respectively. A histogram for homoplasmic 3460G $>$ A detection of pyrosequencing assay was shown as a reference (D).

entire mitochondrial genome was only possible using various individual pyrosequencing reactions on a number of PCR fragments [22]. In this study, we carried out 4 individual PCR amplifications and 4 pyrosequencing reactions ( $40 \mu \mathrm{l}$ of PCR solution) for each sample to achieve the 4 mutation analyses $(11778 \mathrm{G}>\mathrm{A}, 14484 \mathrm{~T}$ $>$ C, 3460G > A and 13708G > A). Obviously, the limits of pyrosequencing method are lower through-put in the SNP mode, larger experimental material consuming and still lab intensive. Instead, our results showed that this biochip format was very flexible and amenable to the specific identification of mtDNA mutations with a single base mismatch and their homoplasmic status in LHON. Detection of all the 32 mutations in LHON took only five hours through all the procedures of PCR amplification and biochip assay. The template preparation was performed in one multiplex PCR reaction $(25 \mu \mathrm{l}$ in each). The main advantage by the biochip over direct sequencing and pyrosequencing was that this simple handling protocol could capture the 32 LHON mutations in mtDNA for massively parallel genotyping in a smaller amount of reaction volume ( $5 \mu \mathrm{l}$ of unpurified PCR solution in each sample) and enable analysis of the mutations in LHON mtDNA at the same time. The biochip results were in full accordance with DNA sequencing and pyrosequencing, proportional to the presence of the homoplasmic mutations $11778 \mathrm{G}>$ A, 14484T $>$ C, 3394T $>$ C and 3460G $>$ A, 13708G $>\mathrm{A}$ and $11719 \mathrm{G}>\mathrm{A}$ or the heteroplasmic $11778 \mathrm{G}$ $>$ A mutation (Fig. 4) in the LHON symptomatic cases and asymptomatic family relatives as well as the absence of all the potential mutations in the control donors, demonstrating that the three methods shared a compatible competency in proving the LHON related mutation sites. 


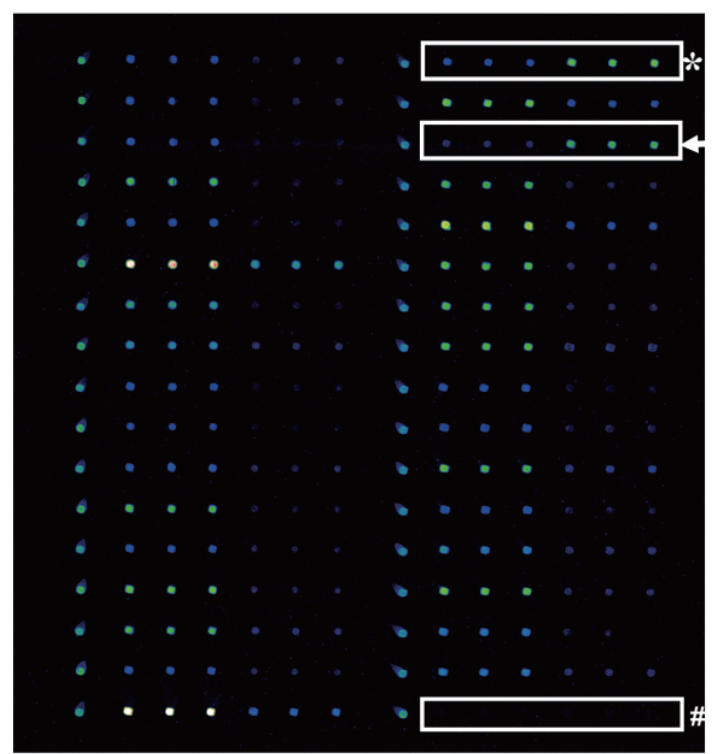

A
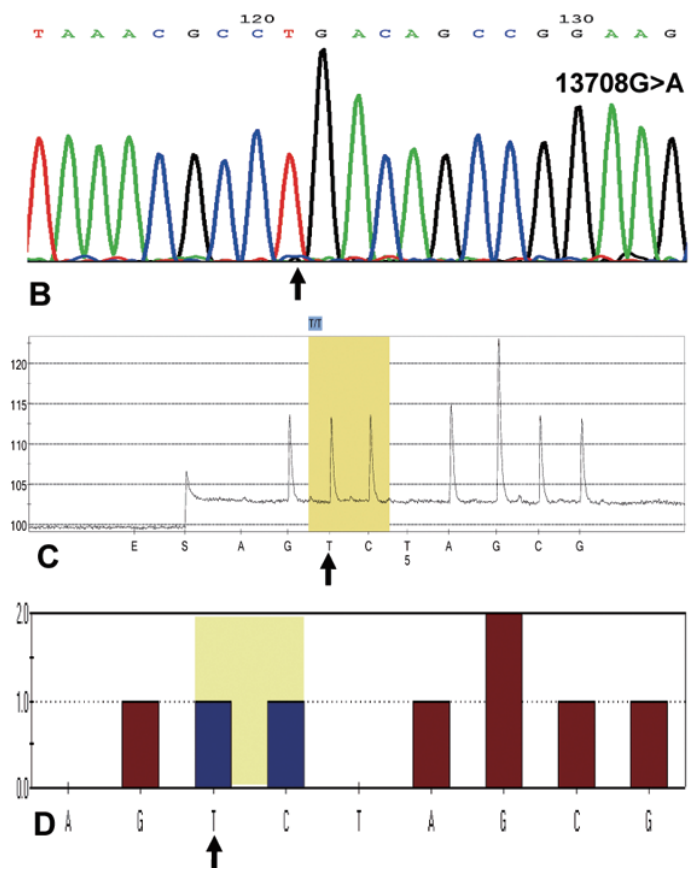

Fig. 6. Detection of 13708 homoplasmic mutation in a patient with LHON by the biochip (A), direct sequencing (B) and pyrosequencing (C). mtDNA mutation 11719 was localized with an asterisk. The wt/mut ratio was 0.08 at the site of mtDNA 13708 by the biochip. A histogram of 13708 homoplasmic mutation of pyrosequencing assay was shown as a reference (D).

\subsection{Clinical analysis of LHON pedigrees}

The diagnosis of LHON usually needs to be confirmed by molecular genetic analysis for one of the three primary mtDNA mutations of LHON: $3460 \mathrm{G}>$ A, 11778G $>$ A and 14484T $>$ C [23]. Kim identified the three mutations in $60(73 \%)$ of the 82 probands of Korean LHON. The frequencies of $11778 \mathrm{G}>\mathrm{A}$, $14484 \mathrm{~T}>\mathrm{C}$ and $3460 \mathrm{G}>\mathrm{A}$ were $56 \%$ (46 cases), $16 \%$ (13 cases) and $1 \%$ ( 1 case), respectively [24]. In this study, we investigated 18 symptomatic cases and 52 asymptomatic relatives from 14 Chinese LHON pedigrees by the biochip method and found that 12 of the patients carried one of the primary mutations $11778 \mathrm{G}$ $>\mathrm{A}, 14484 \mathrm{~T}>\mathrm{C}$ or $3460 \mathrm{G}>\mathrm{A}$, respectively, accounting for $66.7 \%$ (12/18 cases). Of which the mutation $11778 \mathrm{G}>\mathrm{A}$ was prevalent $(55.5 \%, 10$ of 18 patients). The mutations $14484 \mathrm{~T}>\mathrm{C}$ and $3460 \mathrm{G}>$ A occurred in two individual probands $(5.6 \%, 1$ of 18 patients, respectively). It was reported that heteroplasmic frequency of $11778 \mathrm{G}>\mathrm{A}$ in LHON symptomatic cases was lower than that in their asymptomatic maternal relatives, $12.9 \%$ vs. $40 \%$ [25]. In this study, all the mutations of $11778 \mathrm{G}>\mathrm{A}$ in $10 \mathrm{LHON}$ symptomatic cases were homoplasmic, showing $\mathrm{wt} / \mathrm{mut}$ ratio factors of $<0.3(0.01-0.25)$, while 2 of 11 asymptomatic ma- ternal relatives carrying the mutation $11778 \mathrm{G}>\mathrm{A}$ appeared heteroplamic. The ratio factors were 1.11 and 1.34, respectively. The frequencies in the both genotypes accounted for $0 \%$ vs. $18.2 \%$, respectively. These data might partially suggest that the threshold effect of mutants or relative proportions of wild type versus mutated mtDNAs would attribute to the variable clinical features. Furthermore, our biochip data showed that a coexistence of the primary homoplasmic mutation 3460G $>$ A and the homoplasmic mutation 3394T $>\mathrm{C}$ took place in an individual LHON case (Fig. 5). The wt/mut ratios were 0.24 at $3460 \mathrm{G}>\mathrm{A}$ and 0.16 at $3394 \mathrm{~T}>\mathrm{C}$, respectively. Also, the homoplasmic mutation $13708 \mathrm{G}>\mathrm{A}$ alone occurred in a proband with a wt/mut ratio of 0.08 (Fig. 6) and all his asymptomatic maternal members with wt/mut ratios ranging $0.08-$ 0.19 in a typical LHON pedigree, devoid of the three primary mutations. Therefore these results indicated that the mutations 3394T $>\mathrm{C}$ and $13708 \mathrm{G}>\mathrm{A}$ might be involved in LHON pathogenesis by means of aggravating primary mutations in combination or exerting its pathogenic effectiveness alone. The SNP 11719G > A would be a potential genetic bio-barcode of human mitochondrion in China as a result of that it emerged in all the samples tested. In this study, the questionable evidences that five probands presenting typical LHON 
neurological symptoms did not harbor any characteristic mutations described above implied that there would be either an alternative pathogenic mtDNA mutations beyond the panel selected or an overlap case harboring mtDNA mutations that have not yet been identified or a defect derived from unknown nuclear gene and/or other factors contributing to the strikingly different penetrance of LHON. The details need to be investigated further.

\section{Conclusion}

We developed a novel biochip- based genotyping method for detecting and discriminating of single base substitution in mtDNA of LHON and evaluated the feasibility of this biochip format in diagnostics of the entity. By means of a multiplex PCR amplification, thirtytwo mutations were interrogated on one biochip in a single reaction in LHON pedigrees and healthy individuals. The biochip was simple, efficient and reliable. The technique would easily be implemented in clinical applications.

\section{Authors' contributions}

The work presented here was carried out in collaboration between all authors. WDD defined the research theme and designed experiments. GC, HZ, HMC and QHJ carried out the laboratory experiments and analyzed the data. WDD, GC, HMC, XJZ and RFL interpreted the results and prepared the paper. $\mathrm{XCH}, \mathrm{DBC}$, SRH, YML, HYT, XFT, YQW and SS co-worked on associated clinical data collection and their interpretation. WDD and JLZ coordinated the study. All authors have contributed to and approved the manuscript.

\section{Acknowledgements}

This study was supported by a grant of the National Natural Science Foundation of China (30470591) and a grant of the Natural Science Foundation of Anhui Province (090413114) to Dr. W. Du. The authors are thankful to all the patients for their support in this study.

\section{Competing interests}

None.

Ethices approval Ethics approval was provided by the ethics committee for genome research of the Anhui
Medical University of China

\section{Patients consent}

Obtained.

\section{Supplementary material}

Supplementary data can be found at: http://210.45. 96.45/Supplementary/.

\section{References}

[1] P. Barboni, G. Savini, M.L. Valentino, C. La Morgia, C. Bellusci, A.M. De Negri, F. Sadun, A. Carta, M. Carbonelli, A.A. Sadun and V. Carelli, Leber's hereditary optic neuropathy with childhood onset, Invest Ophthalmol Vis Sci 47 (2006), 53035309.

[2] J. Finsterer, Overview on visceral manifestations of mitochondrial disorders, Neth J Med 64 (2006), 61-71.

[3] L.J. Wong, Diagnostic challenges of mitochondrial DNA disorders, Mitochondrion 7 (2007), 45-52.

[4] E.L. Blakely, R. de Silva, A. King, V. Schwarzer, T. Harrower, G. Dawidek, D.M. Turnbull and R.W. Taylor, LHON/MELAS overlap syndrome associated with a mitochondrial MTND1 gene mutation, Eur J Hum Genet 13 (2005), 623-627.

[5] D. Liolitsa, S. Rahman, S. Benton, L.J. Carr and M.G. Hanna, Is the mitochondrial complex I ND5 gene a hot-spot for MELAS causing mutations? Ann Neurol 53 (2003), 128-132.

[6] A. Pandya, X.J. Xia, R. Erdenetungalag, M. Amendola, B. Landa, J. Radnaabazar, B. Dangaasuren, G. Van Tuyle and W.E. Nance, Heterogenous point mutations in the mitochondrial tRNA Ser(UCN) precursor coexisting with the A1555G mutation in deaf students from Mongolia, Am J Hum Genet 65 (1999), 1803-1806.

[7] M.Y. Yen, A.G. Wang and Y.H. Wei, Leber's hereditary optic neuropathy: a multifactorial disease, Prog Retin Eye Res 25 (2006), 381-396.

[8] R.W. Taylor, M.S. Jobling, D.M. Turnbull and P.F. Chinnery, Frequency of rare mitochondrial DNA mutations in patients with suspected Leber's hereditary optic neuropathy, J Med Genet 40 (2003), e85.

[9] T. Pulkes, D. Liolitsa, I.P. Nelson and M.G. Hanna, Classical mitochondrial phenotypes without mtDNA mutations: the possible role of nuclear genes, Neurology 61 (2003), 11441147.

[10] L.J. Wong and R.G. Boles, Mitochondrial DNA analysis in clinical laboratory diagnostics, Clin Chim Acta 354 (2005), $1-20$.

[11] T.G. Drummond, M.G. Hill and J.K. Barton, Electrochemical DNA sensors, Nat Biotechnol 21 (2003), 1192-1199.

[12] V. Alvarez-Iglesias, F. Barros, A. Carracedo and A. Salas, Minisequencing mitochondrial DNA pathogenic mutations, BMC Med Genet 9 (2008), 26.

[13] H.E. White, V.J. Durston, A. Seller, C. Fratter, J.F. Harvey and N.C. Cross, Accurate detection and quantitation of heteroplasmic mitochondrial point mutations by pyrosequencing, Genet Test 9 (2005), 190-199. 
[14] R.G. van Eijsden, M. Gerards, L.M. Eijssen, A.T. Hendrickx, R.J. Jongbloed, J.H. Wokke, R.Q. Hintzen, M.E. RubioGozalbo, I.F. De Coo, E. Briem, V. Tiranti and H.J. Smeets, Chip-based mtDNA mutation screening enables fast and reliable genetic diagnosis of OXPHOS patients, Genet Med $\mathbf{8}$ (2006), 620-627.

[15] S.F. Grant and H. Hakonarson, Microarray technology and applications in the arena of genome-wide association, Clin Chem 54 (2008), 1116-1124.

[16] A. Maitra, Y. Cohen, S.E. Gillespie, E. Mambo, N. Fukushima, M.O. Hoque, N. Shah, M. Goggins, J. Califano, D. Sidransky and A. Chakravarti, The Human MitoChip: a high-throughput sequencing microarray for mitochondrial mutation detection, Genome Res 14 (2004), 812-819.

[17] R.M. Andrews, I. Kubacka, P.F. Chinnery, R.N. Lightowlers, D.M. Turnbull and N. Howell, Reanalysis and revision of the Cambridge reference sequence for human mitochondrial DNA, Nat Genet 23 (1999), 147.

[18] M.C. Brandon, M.T. Lott, K.C. Nguyen, S. Spolim, S.B. Navathe, P. Baldi and D.C. Wallace, MITOMAP: a human mitochondrial genome database-2004 update, Nucleic Acids Res 33 (2005), D611-D613.

[19] W. Du, W. Li, G. Chen, H. Cao, H. Tang, X. Tang, Q. Jin, Z. Sun, H. Zhao, W. Zhou, S. He, Y. Lv, J. Zhao and X. Zhang, Detection of known base substitution mutations in human mitochondrial DNA of MERRF and MELAS by biochip technology, Biosens Bioelectron 24 (2009), 2371-2376.
[20] W. Du, C. Marsac, M. Kruschina, F. Ortigao and C. Florentz, Functionalized self-assembled monolayer on gold for detection of human mitochondrial tRNA gene mutations, Anal Biochem 322 (2003), 14-25.

[21] T. Woehrle, W. Du, A. Goetz, H.Y. Hsu, T.O. Joos, M. Weiss, U. Bauer, U.B. Brueckner and E. Marion Schneider, Pathogen specific cytokine release reveals an effect of TLR2 Arg753Gln during Candida sepsis in humans, Cytokine 41 (2008), 322329.

[22] G.M. Anjum, W. Du, R. Klein, U. Amara, M. Huber-Lang, E.M. Schneider and P. Wiegand, Pyrosequencing-based strategy for a successful SNP detection in two hypervariable regions: HV-I/HV-II of the human mitochondrial displacement loop, Electrophoresis 31 309-314.

[23] P.F. Chinnery, D.T. Brown, R.M. Andrews, R. Singh-Kler, P. Riordan-Eva, J. Lindley, D.A. Applegarth, D.M. Turnbull and N. Howell, The mitochondrial ND6 gene is a hot spot for mutations that cause Leber's hereditary optic neuropathy, Brain 124 (2001), 209-218.

[24] J.Y. Kim, J.M. Hwang, B.L. Chang and S.S. Park, Spectrum of the mitochondrial DNA mutations of Leber's hereditary optic neuropathy in Koreans, J Neurol 250 (2003), 278-281.

[25] W.L. Chuenkongkaew, R. Suphavilai, L. Vaeusorn, N. Phasukkijwatana, P. Lertrit and B. Suktitipat, Proportion of 11778 mutant mitochondrial DNA and clinical expression in a thai population with leber hereditary optic neuropathy, J Neuroophthalmol 25 (2005), 173-175. 


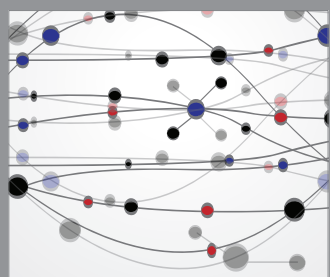

The Scientific World Journal
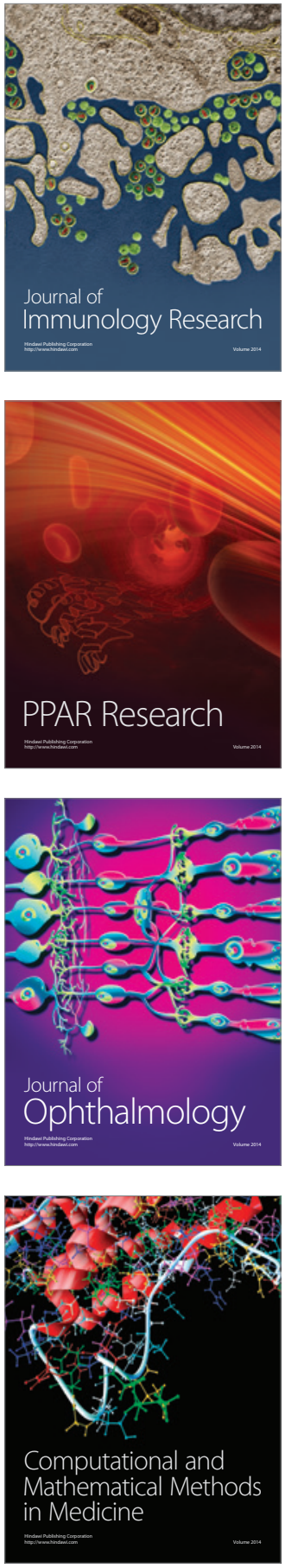

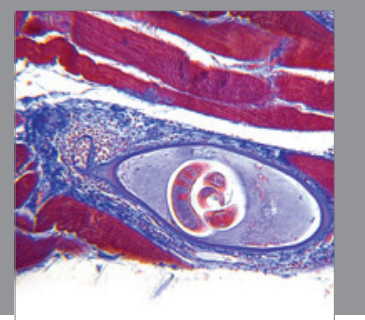

Gastroenterology

Research and Practice
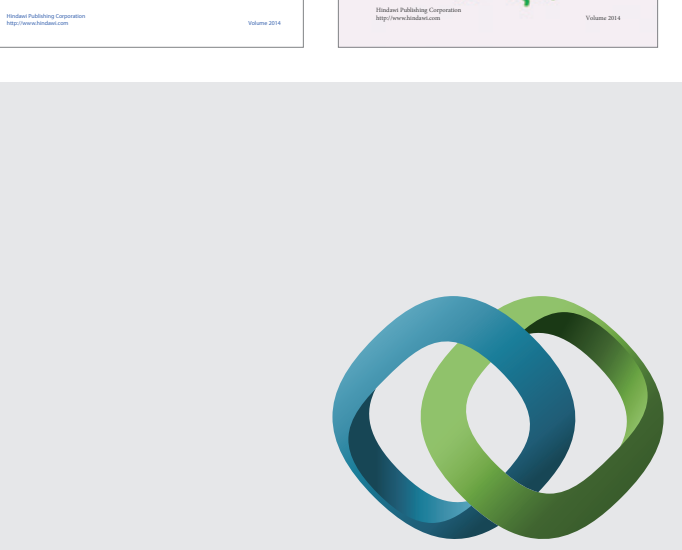

\section{Hindawi}

Submit your manuscripts at

http://www.hindawi.com
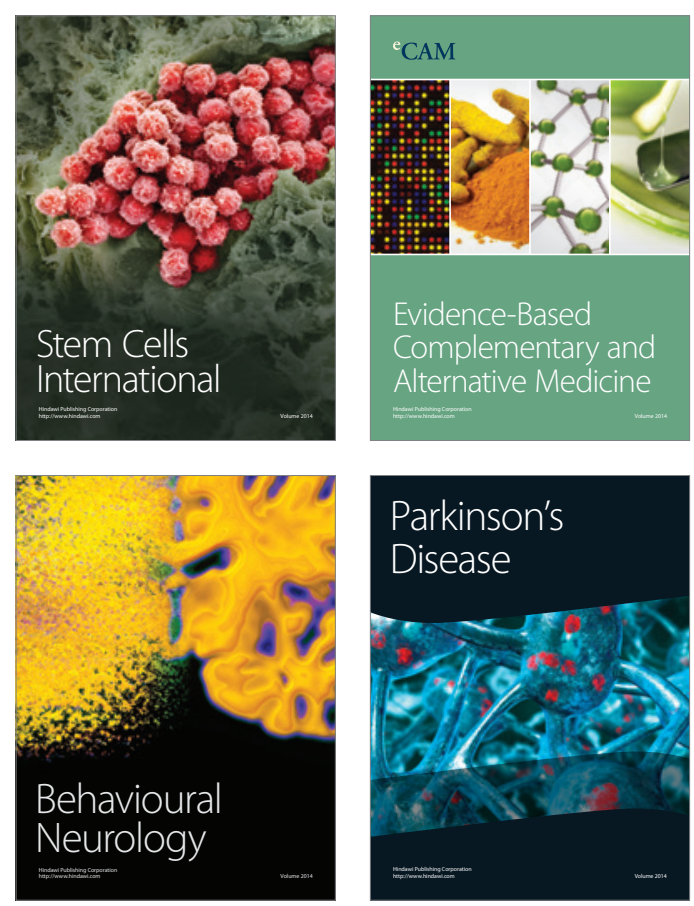

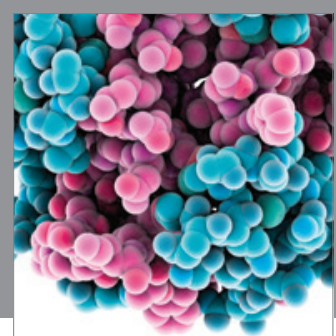

Journal of
Diabetes Research

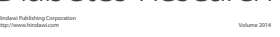

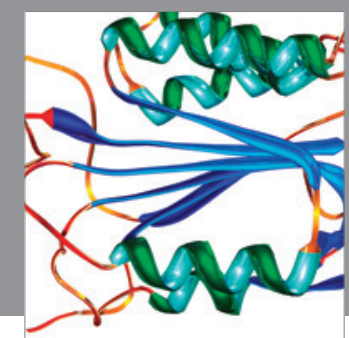

Disease Markers
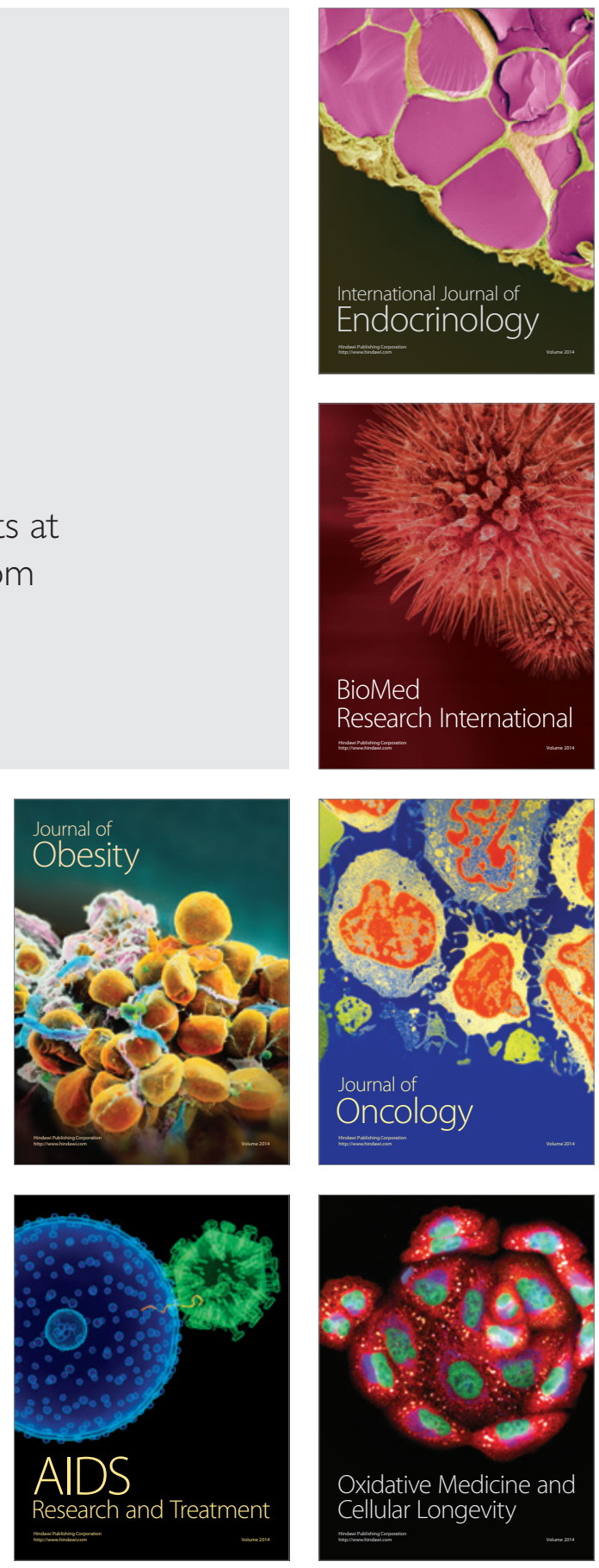